\title{
Characterization of high-power quasi-cw laser diode arrays
}

\author{
Mark A. Stephen ${ }^{* 1}$, Aleksey Vasilyev², Elisavet Troupaki², Graham R. Allan ${ }^{3}$ \\ Nasir B. Kashem ${ }^{1}$ \\ ${ }^{1}$ NASA's Goddard Space Flight Center, Mail Code 694, Greenbelt, MD 20771, USA \\ ${ }^{2}$ Science Systems Applications, Inc., 10210 Greenbelt Road, Suite 600,Lanham, Maryland 20706 \\ ${ }^{3}$ Sigma Space Corporation, 9801 Greenbelt Rd. Suite 103, Lanham MD 20706
}

\begin{abstract}
NASA's requirements for high reliability, high performance satellite laser instruments have driven the investigation of many critical components; specifically, $808 \mathrm{~nm}$ laser diode array (LDA) pump devices. Performance and comprehensive characterization data of Quasi-CW, High-power, laser diode arrays is presented.
\end{abstract}

Keywords: laser, diode, array, diode-pumped, space qualified, reliability, characterization, quasi-constant wave (QCW),

\section{INTRODUCTION}

Laser remote sensing instruments have been promising to revolutionize data collection for many years but instruments collecting large-scale data sets have proven elusive, this is in part, due to the complexity and cost of the system. A major shortcoming of many lidar instruments is the inability to run in a reliable, continuous and autonomous mode. Over the years, substantial effort has been devoted to pushing the state-of-the-art for new and better measurements but very few instruments reach a "turn-key" state. This is a problem of particular interest to NASA where we desire to deploy laser -based instruments on satellites which are required to operate continuously for years.

In order to address many of the reliability concerns, NASA has initiated a program to understand and improve the reliability of laser instruments and associated components. Many of NASA's recent laser based instruments including GLAS [1], CALIPSO [2], and MLA [3] have employed diode-pumped Nd:YAG lasers. Several near-term missions under consideration propose using the same technology. The diode lasers are operated in quasi-CW(QCW) mode. Reliability of Quasi-CW, $808 \mathrm{~nm}$, laser diode arrays (LDAs) used in these instruments is of particular interest as the LDA's performance is critical to the operation of the laser system. The arrays are potentially a single point failure for the laser. If the arrays fail, the performance of the laser will degrade and jeopardize the mission. As a result the LDAs are a being investigated to help improve the reliability of laser instruments in future missions.

QCW laser diode arrays have a unique set of challenges that must be understood and addressed in order to improve their functionality in continuously operable lasers. Because the arrays are the power source for the laser, their correct operation is critical to the performance of the entire instrument. QCW operation puts thermo-mechanical strain on the package that makes it fundamentally different than $\mathrm{CW}$ devices. For many applications, billions of pulses are required which means billions of thermal cycles. Temperature has been identified as an important parameter in the performance of a diode lasers with the lifetime decreasing exponentially with increasing temperature [4] and repeated temperature cycles induced by current pulses contributing to degradation over time [5]. Because of its importance, we use several techniques to monitor the temperature of the device.

LDAs are complex devices with multiple failure mechanisms which are not only dependent on operational conditions, but on the design, manufacture and operational history of the device, so modeling reliability is difficult. In addition, different failure mechanisms will have different activation conditions and finding accurate acceleration parameters for testing is not straightforward. Statistics for performance are expensive due to the cost of the arrays. Designs, materials,

• cmail: mark.a.stephen@nasa.gov, phonc: 301-614-6737 
procedures can change in an effort to improve device performance that can degrade (or negate) the statistics collected on previous devices. The QCW array market does not appear to support the kind of performance and reliability testing that is justified in the telecom market where it is possible to require adherence to stringent Telcordia standards. The competitive nature of the business means the vendors and designs change. In addition to smaller market size, the operational conditions and requirements are also much more diverse so it is difficult to address all QCW pump arrays with a single product. So the target is further fractured making it an even smaller effective market. It is difficult to correlate how the arrays will operate under one set of conditions based on testing from a different set of conditions. NASA's Goddard Space Flight Center has an intensive program to investigate the reliability concerns associated with QCW, $808 \mathrm{~nm}$, high-power, laser diode arrays optically pumping laser-based instruments.

\section{LASER DIODE ARRAY CHARACTERIZATION AND TESTING}

From the engineering and physics of failure mechanisms in LDAs we have developed a state-of-the-art characterization facility to measure important parameters for the evaluation of array performance and reliability. We have developed long-duration tests ${ }^{*}$ to evaluate the performance of the LDAs under various operating conditions. We also track key characteristics throughout the life of the arrays to try and identify both failure mechanisms and their precursors. In this way we hope to develop evaluation criteria to screen for devices that exhibit a high-risk of failure and operating conditions that have a high-risk of inducing failure.

This information has already enabled NASA to improve its diode selection through better specification of device parameters and construction. We have also been sharing this information with the suppliers.

In developing these tests we have devoted substantial effort into generating repeatable and safe handling procedures so we do not adversely affect the arrays. The lab environment uses air filtration and electro-static discharge (ESD) precautions. The device under test is attached to a test plate from which all mechanical and electrical contacts are made, minimizing handling of the LDA. This test plate is then attached to the various stations for testing. Grounding plugs are used as necessary.

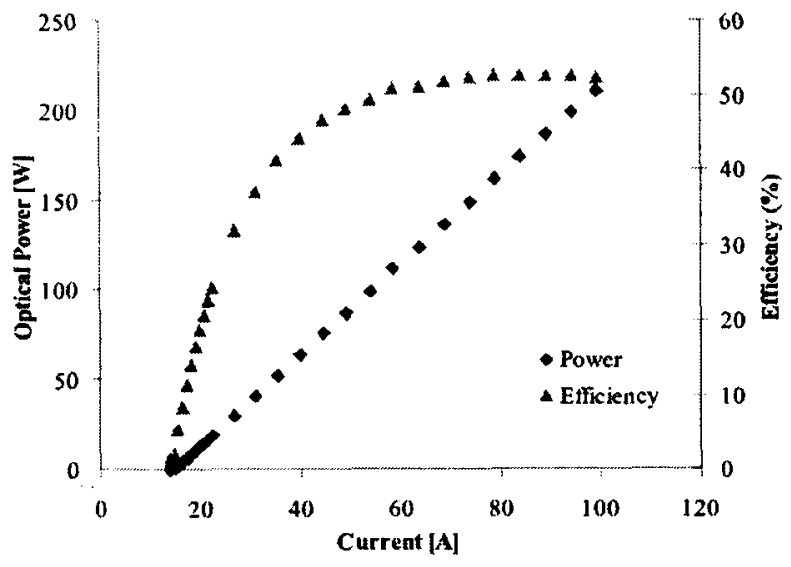

(a)

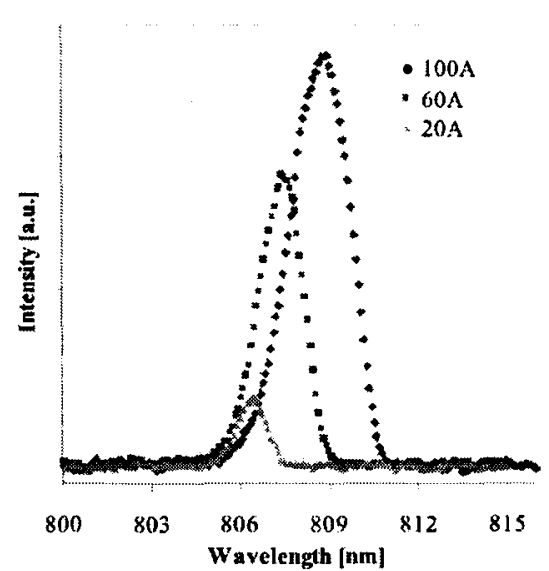

(b)

Figure 1: These graphs show typical performance of (a) average optical power and efficiency vs. drive current and (b) average spectrum at three different drive currents of a conductively cooled 2-bar laser diode array.

\footnotetext{
"We deliberately avoid the term "life test" in this context because we question the statistical validity of predicting the future performance of similar products from the results. If the results prove to be repeatable over time and the product processes stabilize, the tests will earn more confidence as predictors of performance.
} 
The characterization measurements include: optical power measurements (spatially and temporally averaged, spatially resolved, polarization resolved and temporally resolved); electrical parameters (voltage, drive current and efficiency); thermal profiling (temporally resolved and spatially resolved surface imaging and modeling); spectral measurements (spatially and temporally averaged, time-resolved spectroscopy and spatially resolved spectroscopy); and facet microscopy (near, dark field, nomarski, extended focal imaging and scanning electron microscopy). The LDAs are, in general, pumped with near-square current pulses of $200 \mu$ s duration at repetition rates up to $100 \mathrm{~Hz}$ yielding duty cycles of $\leq 2 \%$.

The data in figure 1 shows the most basic characteristics of device performance. Plotted in (a) is the optical power as a function of peak drive current and in (b), the spectral content at three different drive currents. These power and spectral measurements use an integrating sphere to spatially average the LDA's emission. The efficiency is calculated from the optical power, drive current and voltage across the array.

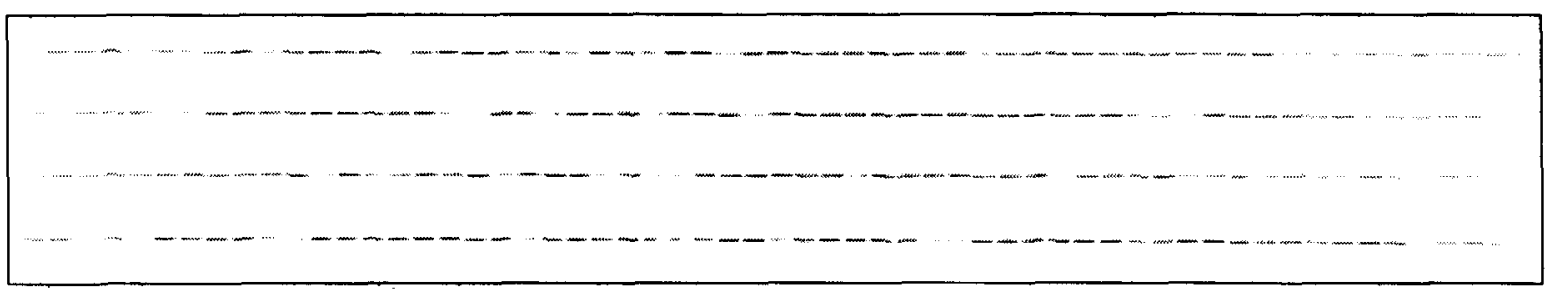

(a)

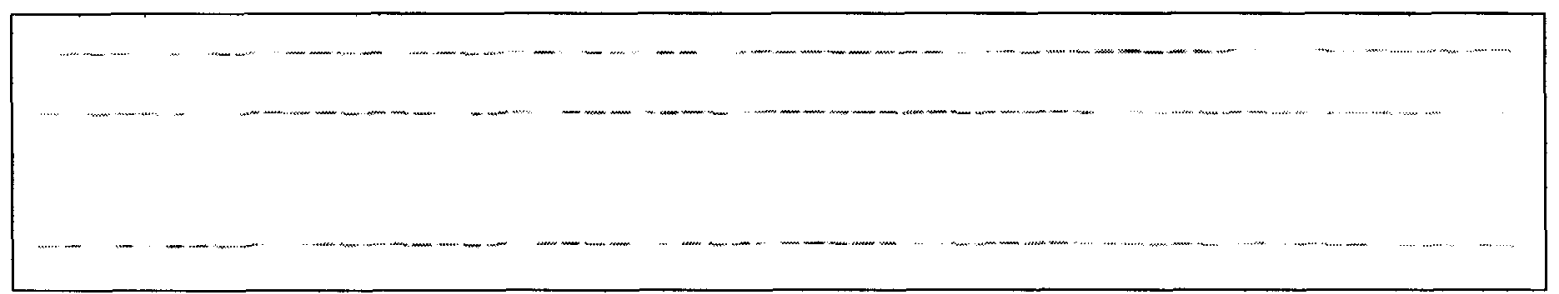

(b)

Figure 2: Near field negative-images of a 4-bar laser diode array captured with a Si-CCD camera, each dark "dash" corresponds to an emmitter. The darker the dash the more intense the emission. Image (a) shows the initial inspection of the array when received by NASA and after vendor "burn-in". Image (b) shows a subsequent inspection after the array had operated for 120 million shots. It clearly shows that one bar has failed completely and degraded performance from other emitters.

A monochrome Si-CCD camera is used to capture the uniformity of the emitter array, see figure 2 above. These are near-field images of a 4-bar laser diode array where each dark "dash" corresponds to an emitter: the darker the dash, the more intense the emission. Image (a) shows the initial inspection of the array. This picture was recorded after vendor "burn-in" and documents the base-line performance of the device when received by NASA. Image (b) shows a subsequent inspection after one hundred and twenty million shots. It clearly shows that one of the four bars has failed completely and degraded performance from other emitters. By resolving the polarization of the emitted light in this optical system, one may be able to measure stress induced changes in the diode bar. Polarization-resolved and spatially resolved inspection of the emitted light has the potential to show new and changing stress points in the array, revealed through stress induced bi-refringence. In this measurement a polarizer is placed in front of the CCD camera and the results recorded, see figure 3.

Figure $3 \mathrm{a}$ and $3 \mathrm{~b}$ show spatially-resolved and polarization-resolved power measurements superimposed on the thermal profile of a single bar of an array. The blue line shows relative optical power polarized parallel to the fast axis of the emitters. The pink line shows the relative power polarized parallel to the slow axis of the emitters across the same bar. Because the light is preferentially polarized in the fast axis, the pink line is scaled by $\sim 200 \mathrm{X}$. The yellow line shows the relative temperature profile across the same part of the array. The left portion of figure 3-a shows a de-polarization correlated to localized bar heating. In figure 3-b, there are de-polarization effects not correlated to heating so we think 
this may give us a new tool to investigate the LDAs. The normal de-polarization ripples shown along the bar are correlated with the v-grooves which separate the emitters. This supports the idea that the de-polarization in the spatially resolved output indicates stress points.

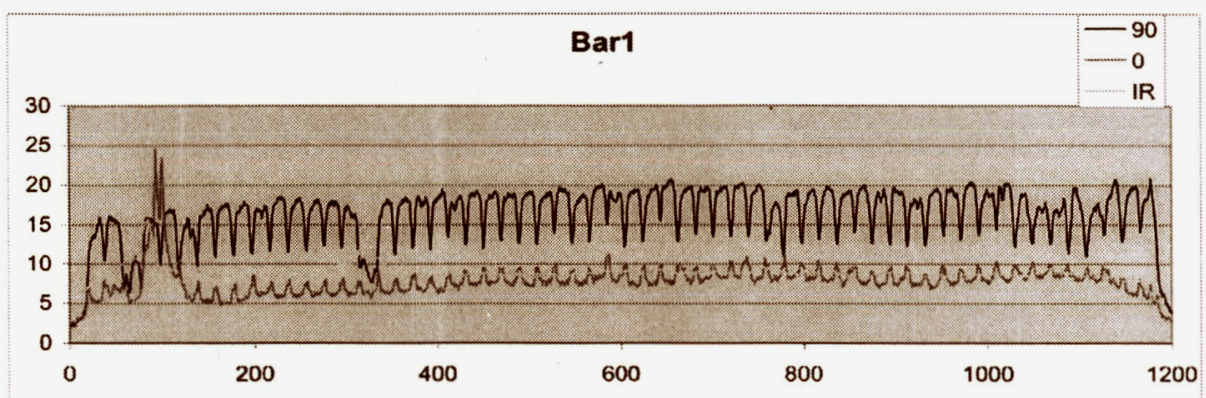

(a)

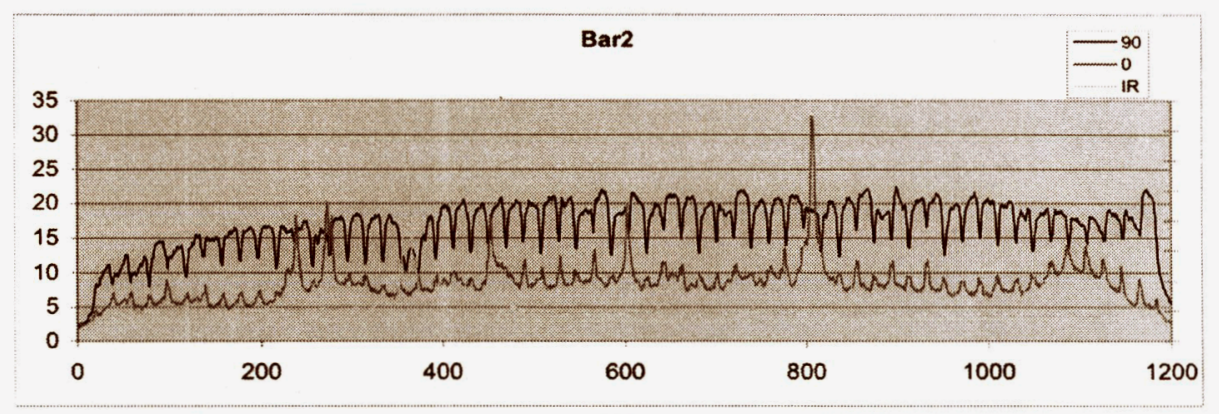

(b)

Figure 3: These graphs show spatially resolved polarized-power measurements superimposed on the thermal profile. The blue line shows relative power polarized parallel to the fast axis of the emitters. The pink line shows the relative power, polarized parallel to the slow axis of the emitters across the same bar. Because the light is preferentially polarized in the fast axis, the pink line is scaled by $\sim 200 \mathrm{X}$. The yellow line shows the relative temperature profile across the same part of the array. (The scale across the horizontalaxis is $\sim 1 \mathrm{~cm}$ corresponding to the bar length.)

As temperature is an important parameter we use several techniques to monitor this parameter in addition to the operational temperature set-point. The induced temperature rise within the current pulse is easily measured optically and can be calculated from the wavelength chirp induced by the bulk heating of the semiconductor. This technique gives the temperature rise within the active region of the LDA. We have developed two techniques to measure the spectral output of the LDA; one which looks at the spatially average but temporally resolved output of the LDA and one which is spatially resolved but temporally averaged.

Simply by inserting a grating in the optical path of the CCD imaging system one can both spectrally-resolve and spatially-resolve the output. Currently this technique temporally averages the output of each emitter of the LDA. Figure 4 is a spectrally resolved near-field image of a 4-bar laser diode array. As in figure 2, each dark feature in figure 4 corresponds to the intensity of the light emitted by each element, however the vertical distribution indicates the temporally averaged spectral distribution of light within the pulse. The vertical distribution indicates the wavelength dispersion. Within each bar, the vertical position corresponds to the time averaged peak wavelength. Different peak wavelengths for different emitters can be caused by local hot spots or stress in the diode bar. 


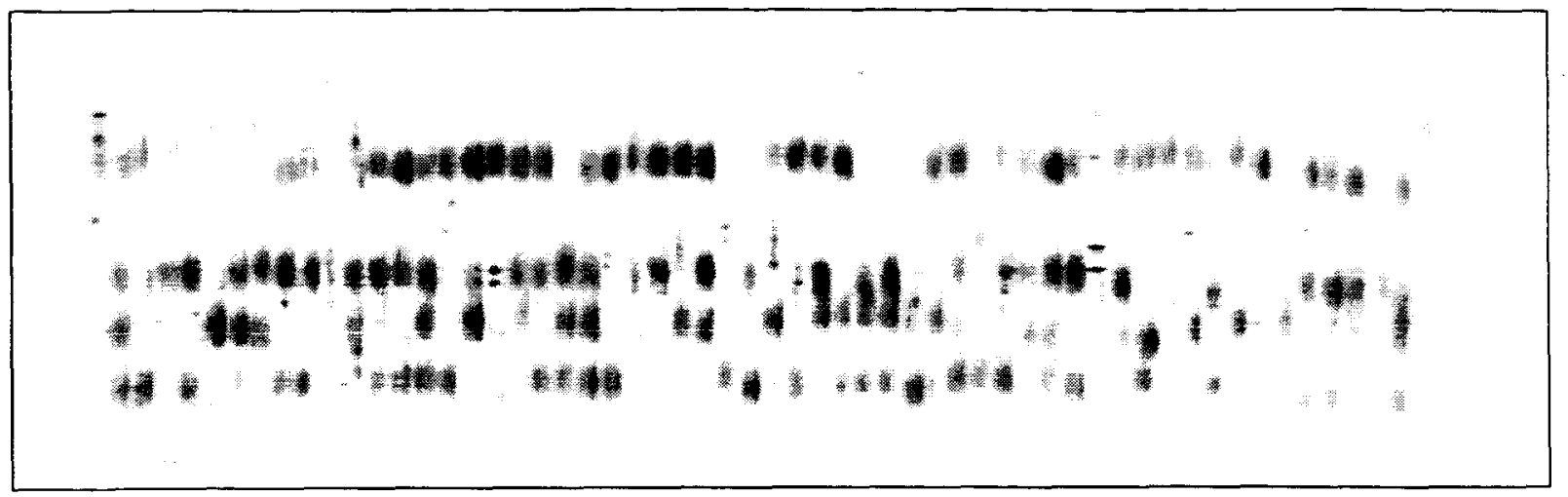

Figure 4: Spectrally resolved and spatially resolved output of the grating imager. As in figure 2, the darkness of the feature corresponds to the intensity of the light. The vertical distribution indicates the wavelength dispersion of each emitter.

The spatially averaged but temporally resolved temperature change within a device is also measured. We measure the wavelength chirp of the output during the $200 \mu$ s drive pulse. From this data we can then calculate the temperature rise in the active region during the pulse. As shown in figure 5, a $6^{\circ} \mathrm{C}$ temperature rise is induced from a $70 \mathrm{~A}, 200 \mu \mathrm{s}$ current pulse. Thus every puise induces a temperature cycle stressing the device. More details on this measurement and analysis can be found in [6].

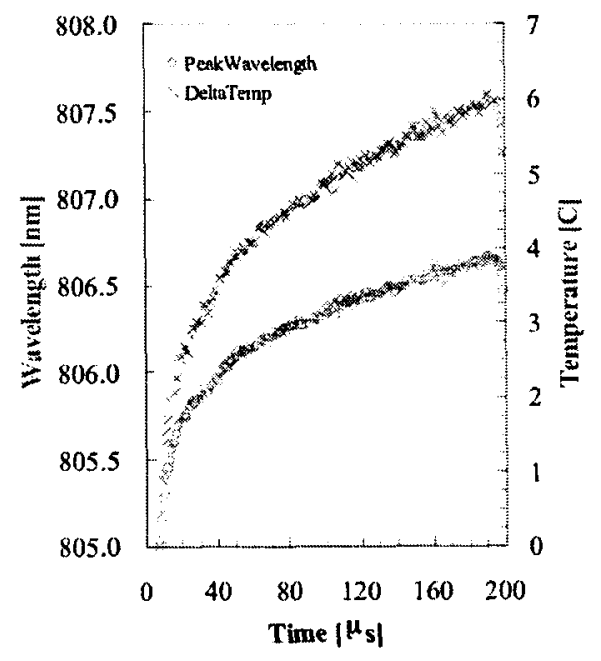

Figure 5: This graph shows the change in peak emission wavelength and temperature of an LDA during a $200 \mu$ sump pulse.

In addition to the emission measurements a complete visual microscopic inspection is performed. A front facet inspection includes a 50X dark field image and 50X and $200 \mathrm{X}$ bright field images and a bright field microscopic inspection of the side of the array at both $50 \mathrm{X}$ and $200 \mathrm{X}$. Additional capabilities include scanning electron microscopy (SEM), nomarski imaging, and extended focal imaging if the initial inspection warrants further investigation. Examples of some of these techniques are illustrated in the appendix.

The thermal properties of the LDAs are also critical to device performance and several techniques have been developed to measure the temperature of the device. A powerful technique to determine the temperature change of the devices is infra-red (IR) imaging. We use a camera sensitive in the 3-5 $\mu \mathrm{m}$ range to measure thermal profiles. In figure 6-a, we show a normal thermal profile illustrating an LDA with small temperature gradients. In figure 6-b, an array with 
localized hotspots is shown. Note the difference in scales between the two figures. Often, even with very high temperature gradients, the output power and spectrum can retain nominal values. So this measurement gives insight into the device not available by other means. We also adjust the exposure and trigger times to get a time series thermal profile. In this case we can measure the thermal dynamics during a current pulse. More details on this measurement and associated modeling can be found in [7]. An example of a time series is shown in Figure 7. It demonstrates the evolution of hotspots within a current pulse. Using these measurements we can increase our understanding of the package in general but also have tools to compare different packages to try to judge the best device.

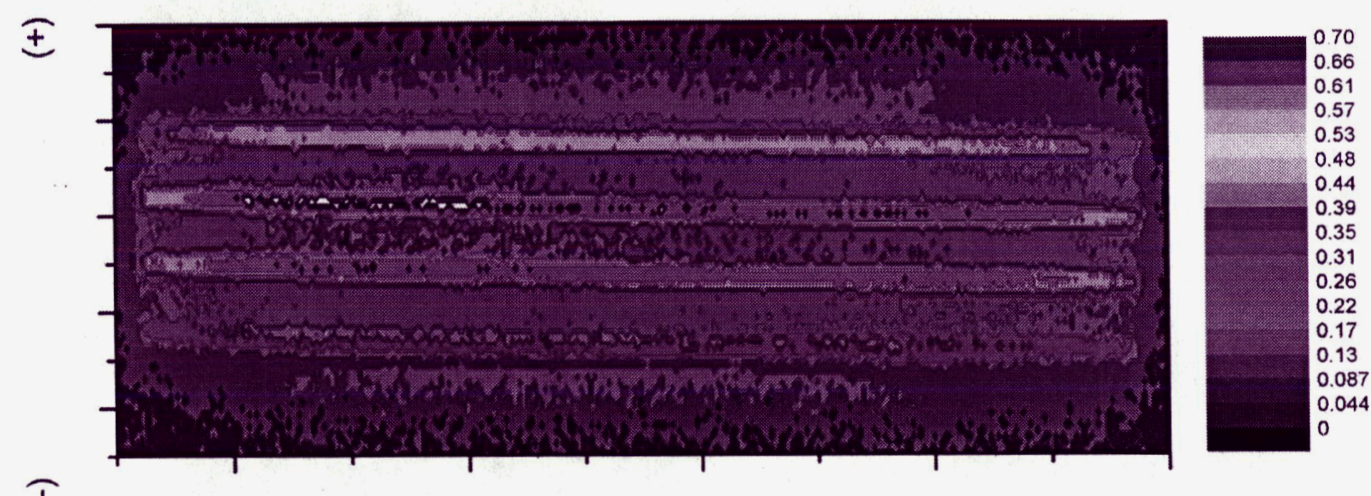

(a)

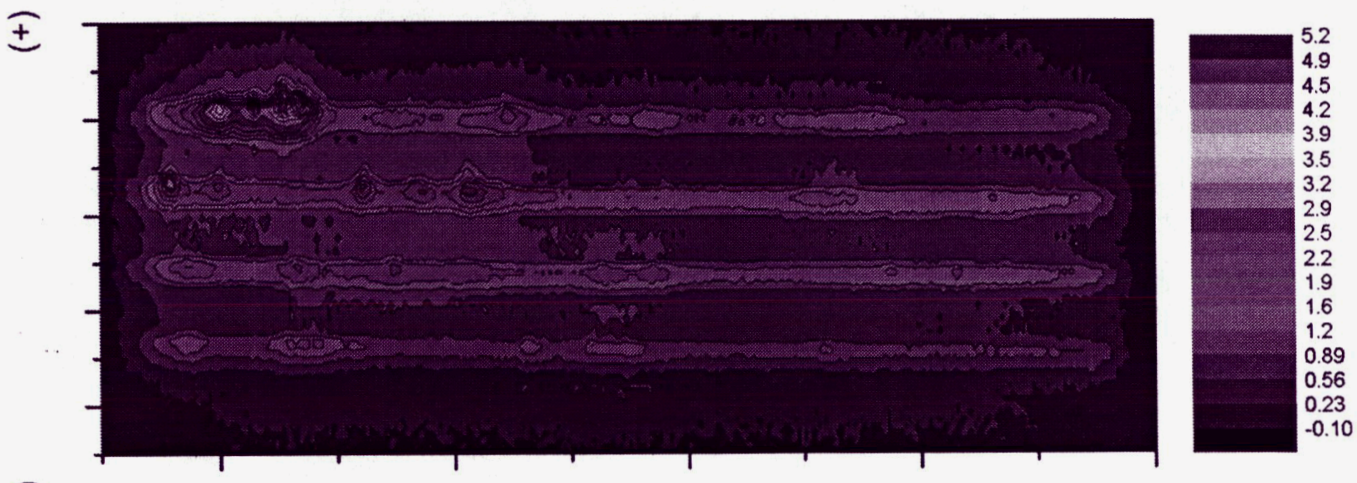

I

(b)

Figure 6: Thermal IR imaging of two 4-bar laser diode arrays showing in one case (a) an even temperature distribution across the bars and in (b) a thermal profile showing localized hot spots. Note the difference in the temperature scales. 


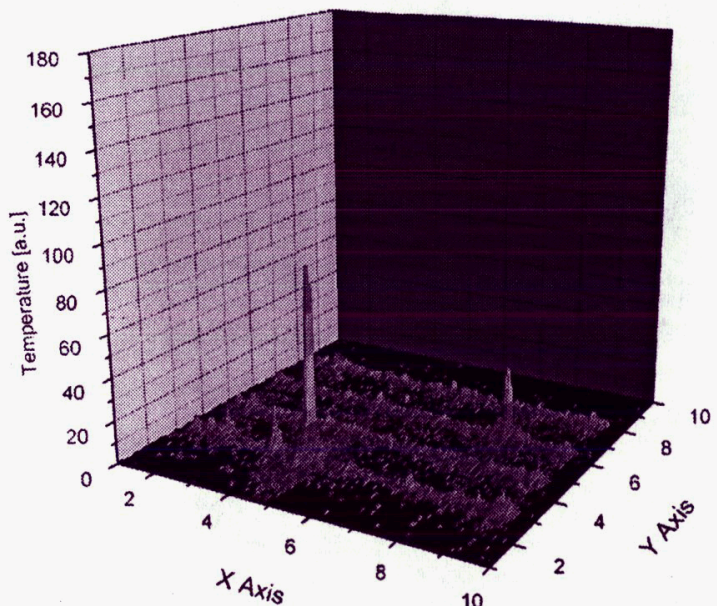

(a)

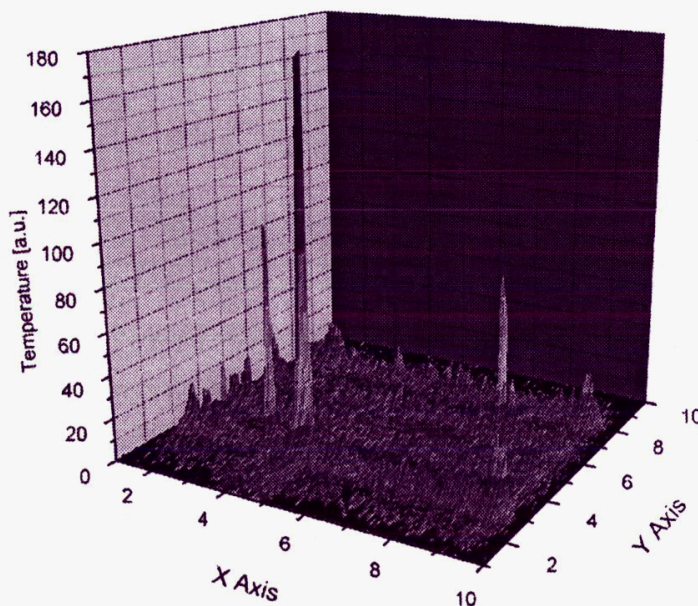

(b)

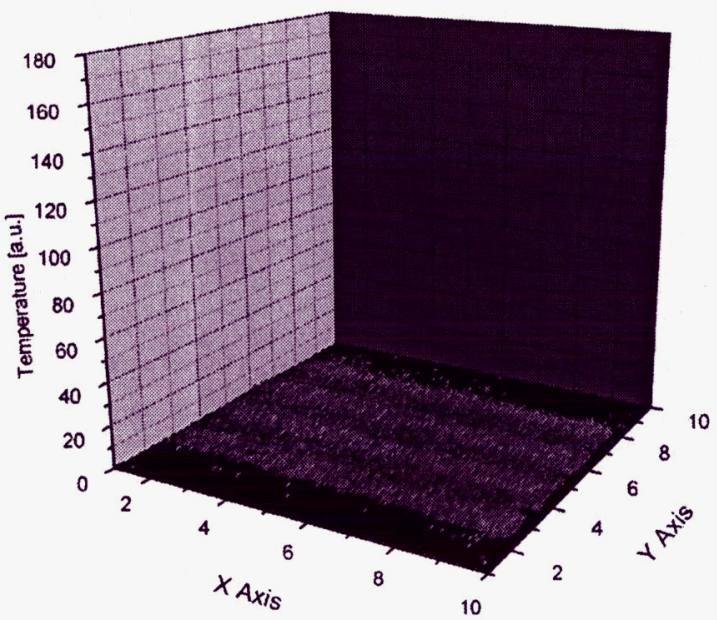

(c)

Figure 7: Time series of IR image showing the evolution of hot spots. Part (a) shows the array $50 \mu$ s into the pump pulse; part (b) shows the array $200 \mu \mathrm{s}$ into the pump pulse; and (c) shows the array $1350 \mu \mathrm{s}$ after the end of the pulse.

\section{PERFORMANCE TEST RESULTS}

In addition to the characterization measurements, we have also begun to operate a number of arrays in long-duration tests. We are trying to accomplish several things with these tests. This will establish an operational lifetime for the LDAs. As we build a database, we will be able understand operational modes that can improve reliability. We want to gain insight into the failure mechanisms as they occur. By understanding these mechanisms, we may identify precursors to damage.

In the first test, we mimick the expected thermal environment for MLA [2]. We operated 12 LDAs (8 4-bar arrays and 4 2-bar arrays) in a matrix where some arrays were power cycled, some were temperature cycled, some were both power and temperature cycled and controls were operated continuously at constant temperature. Both cycles happened over one-hour periods. The power cycled arrays were turned on for 30 minutes and then off for thirty minutes. The 
temperature-cycled arrays were heated linearly $10^{\circ} \mathrm{C}$ over ten minutes and cooled linearly for ten minutes with 20 minutes of settling between each change. Results of this test are shown in Figure 8. Array D12, had a bar failure at 10 million shots and completely failed after $\sim 150$ million shots. The remainder of the arrays continued to operate with little or no degradation after nearly two years of continuous operation at a $30 \mathrm{~Hz}$ repetition rate.
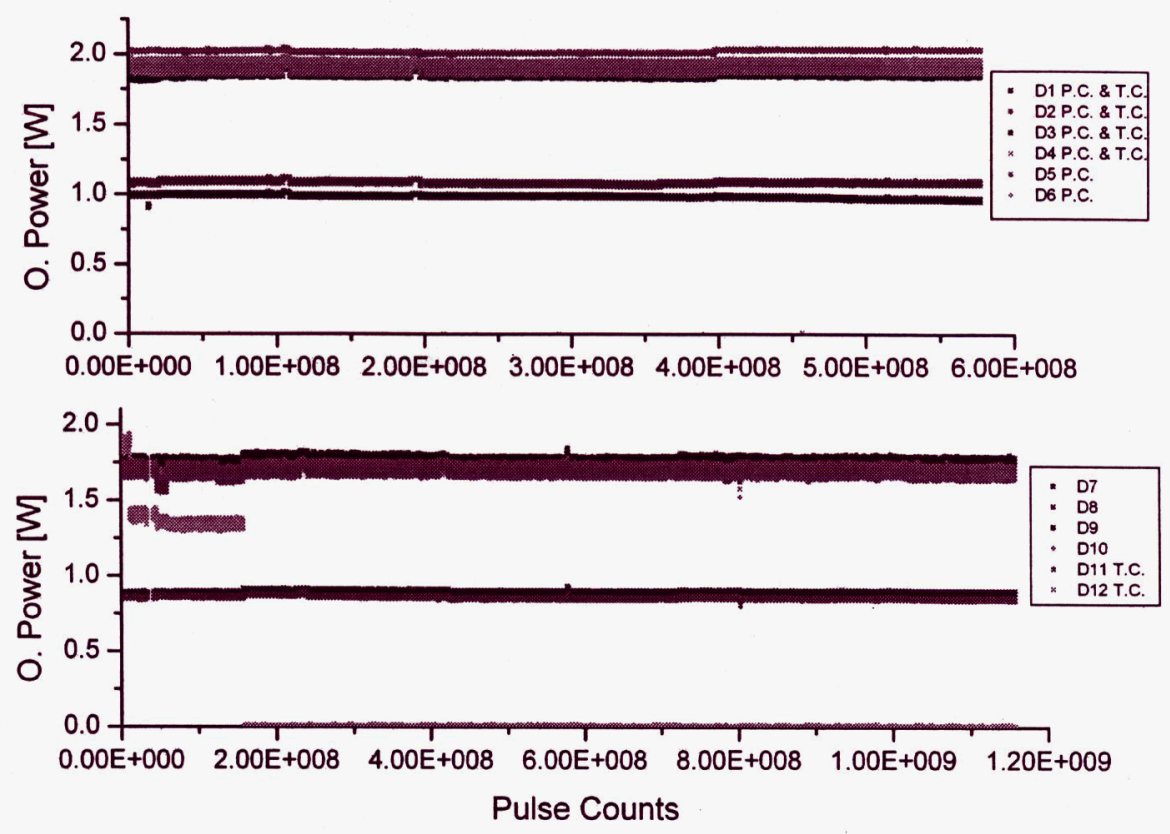

Figure 8 - Performance of (above) power cycled LDAs operated for almost 0.6 billion pulses and (below) constant power LDAs operated for nearly 1.2 billion pulses. Arrays on the legend labeled "T.C." were temperature cycled. These LDAs were tested at 100 Amps peak drive current at $200 \mu$ s pulse width and $30 \mathrm{~Hz}$ repetition rate.

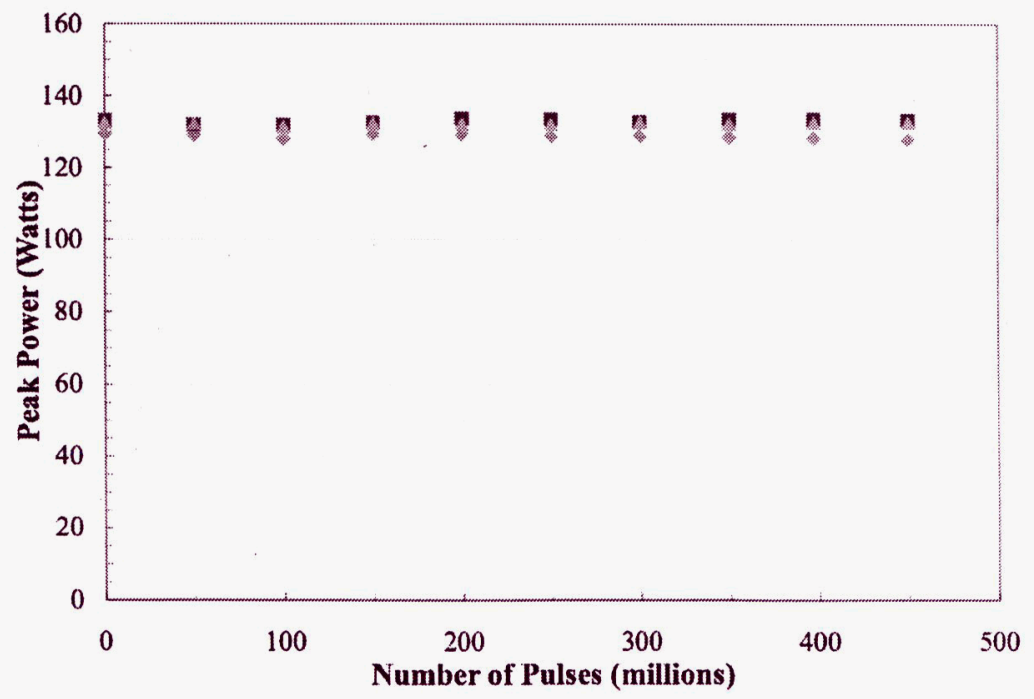

Figure 9 - Test results of three 2-bar LDAs operating with a $200 \mu$ s pulse width, a $100 \mathrm{~Hz}$ repetition rate and a 70 Amp current 
We are also running two tests with a 2 percent duty cycle: one with $200 \mu$ s pulse width and $100 \mathrm{~Hz}$ repetition rate, the other with $80 \mu$ s pulse width and $242 \mathrm{~Hz}$ repetition rate. The results of the tests, to date, are shown in figures 9 and 10 . These tests will not only show how the devices will perform, but also give us the opportunity to judge the relative importance of our measurements. For example, hot spots from the thermal profile are a possible screening criterion. However the threshold for how hot it has to be to cause a problem is not well understood. There are a number of measurements with potential to predict problems with an LDA but we need to quantify their importance. It is necessary to correlate the performance and durability of LDAs with the results of the initial and subsequent characterization measurements. This could lead to screening criteria for the evaluation of LDAs.
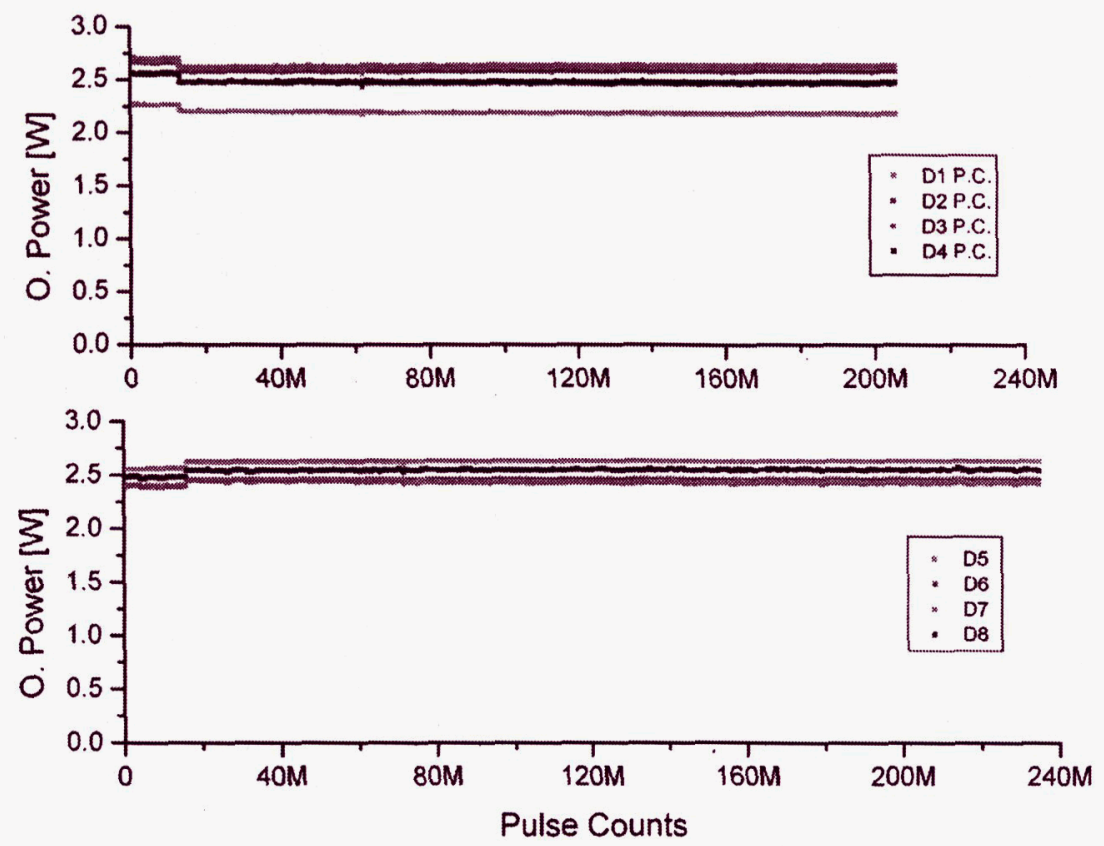

Figure 10 - Test results of eight 4-bar LDAs operating with an $80 \mu$ s pulse width, a $242 \mathrm{~Hz}$ repetition rate and a 50 Amp current.

\section{CONCLUSIONS AND FUTURE WORK}

Although alternate laser technologies and architectures (e.g. fiber lasers and amplifiers) hold great potential for many future applications, diode pumped Nd:YAG lasers continue to be advantageous when high peak powers and high pulse energies are required. By addressing the component technology, in particular the pump diodes, the reliability and performance issues can be resolved. To this end, we have developed state-of-the-art infrastructure to measure LDA performance and characteristics so we can gain a better understanding of failure rates, failure mechanisms and the effects of operating conditions. We have described an array of measurement capabilities: optical power, spectra, electrical drive parameters, thermal profiles and array heating. We have developed additional measurements such as depolarization output to measure device stress. Comprehensive microscopic inspections help track changes in the physical appearance of the LDAs. We are currently adding a photoluminescence apparatus to help detect stress and defects. Array performance in satellite environmental conditions such as radiation, vibration, vacuum and thermal stressing is also considered [8]. Our research results and facilities are being applied to the design of future missions to help mitigate the potential risks of deploying diode-pumped lasers. Collaboration with the LDA vendors is also a key factor in the success of these programs. 


\section{REFERENCES}

[1] H. J. Zwally, B. Schutz, W. Abdalati, J. Abshire, C. Bentley, A. Brenner, J. Bufton, J. Dezio, D. Hancock, D. Harding, T. Herring, B. Minster, K. Quinn, S. Palm, J. Spinhirne, R. Thomas, "ICESat's laser measurements of polar ice, atmosphere, ocean, and land" in Journal of Geodynamics 34 (3-4): 405-445 Oct-Nov 2002

[2] Sun, X., Cavanaugh, J. F., Smith, J. C., Bartels, A. E.; "Design and Performance of the Mercury Laser Altimeter" in Conference on Lasers and Electro-Optics, 2004

[3] D. M. Winker, W. Hunt, C. Hostetler, "Status and performance of the CALIOP lidar", Proceedings of SPIE - Laser Radar Techniques for Atmospheric Sensing, Vol. 5575, pp. 8-15, 14 September 2004

[4] Mitsuo Fukuda, Reliability and degradation of semiconductor laser and LEDs, Boston, MA: Artech House, c1991. ISBN 0890064652.

[5] A. Gerhardt, F. Weik, Tien Quoc Tran, J. W. Tomm, T Elsaesser, J. Biesenbach, H. Muntz, G. Seibold and M. L. Biermann, "Device deformation during low-frequency pulsed operation of high-power diode bars". Applied Physics Letters, V84, Number 18, pp 3525-3527, 3 May 2004

[6] Alex Vasilyev, Graham R. Allan, John Schafer, Mark A. Stephen and Stefano Young, "Optical \& Thermal Analyses of High-Power Laser Diode Arrays", Technical Digest, Seventeenth Solid State and Diode Laser Technology Review, p-38, 8-10 June, 2004 Albuquerque, New Mexico.

[7] Graham R. Allan, Aleksey Vasilyev, Elisavet Troupaki, Nasir Kashem and Mark A. Stephen. "Time-Resolved Optical \& Thermal Analyses of High-Power Laser Diode Arrays", Proceeding of the 2005 Earth-Sun System Technology Conference, ESTC-2005, B3P2, 28-30 June 2005, College Park, Maryland.

[8] Elisavet Troupaki, Nasir B. Kashem, Graham R. Allan, Aleksey Vasilyev and Mark Stephen, "Space Qualification of Laser Diode Arrays", Technical Digest, eighteenth Solid State and Diode Laser Technology Review, p38, 8-10 June, 2005 Los Angeles, California. 


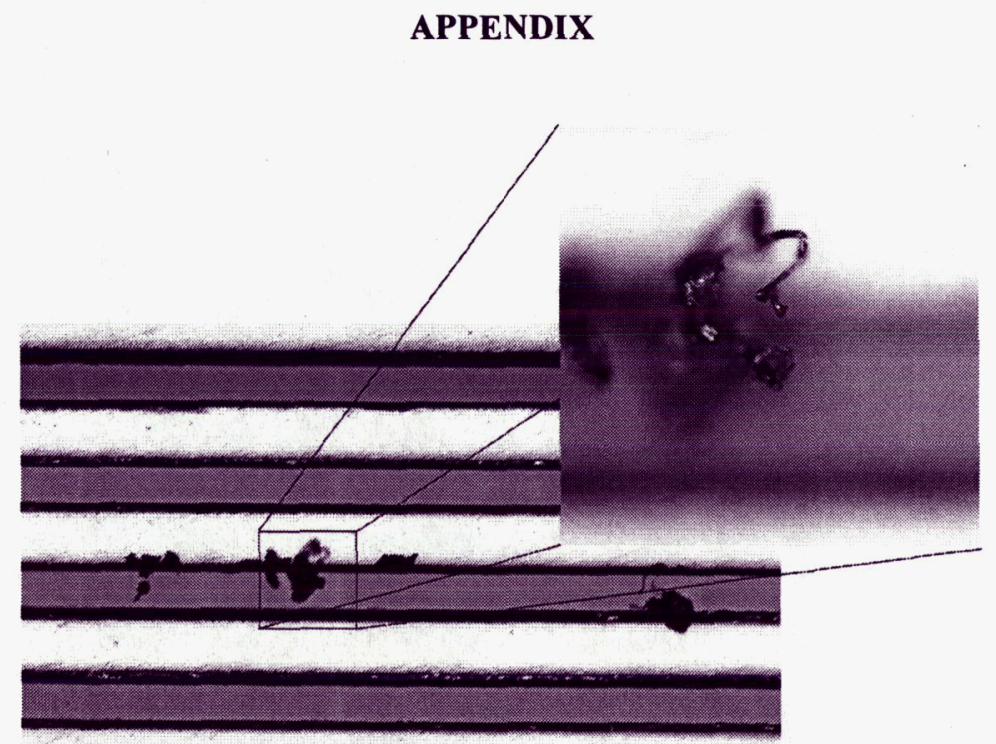

Figure A-1: This shows a 50X bright field microscope image of a 4-bar LDA with a 200X zoomed-in section of a portion of interest The array depicted is D12 of Figure 8. It was taken after 120 million shots were accumulated - between the bar failure and the array failure. The image shows interesting solder structure as well as illustrating the limitations in depth of field.

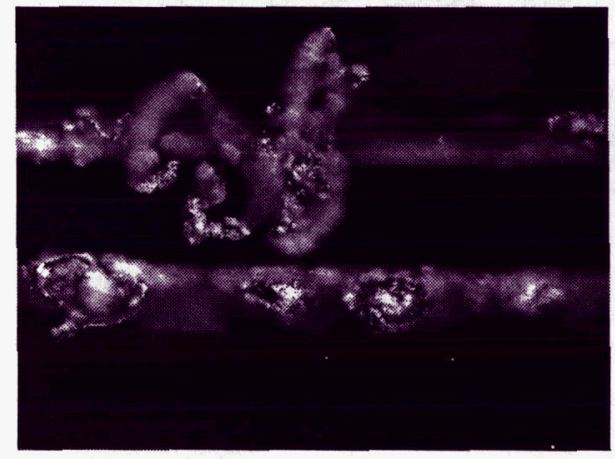

(a)

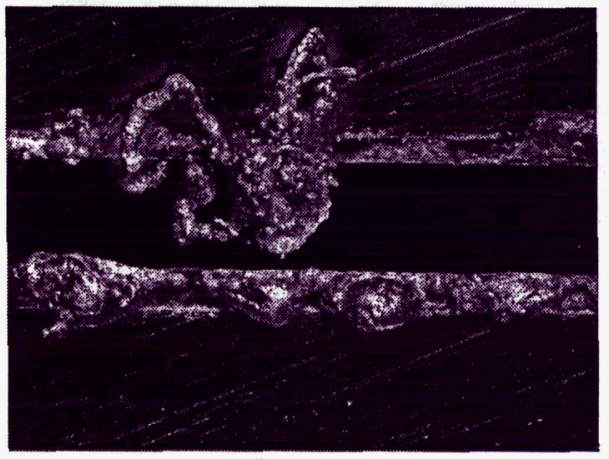

(b)

Figure A-2: This shows the same portion section of the LDA as Figure A-1 but with a 200X Dark field image. In (a) a standard image is shown and in (b) the extended focal imaging technique is applied to capture more depth of field. 


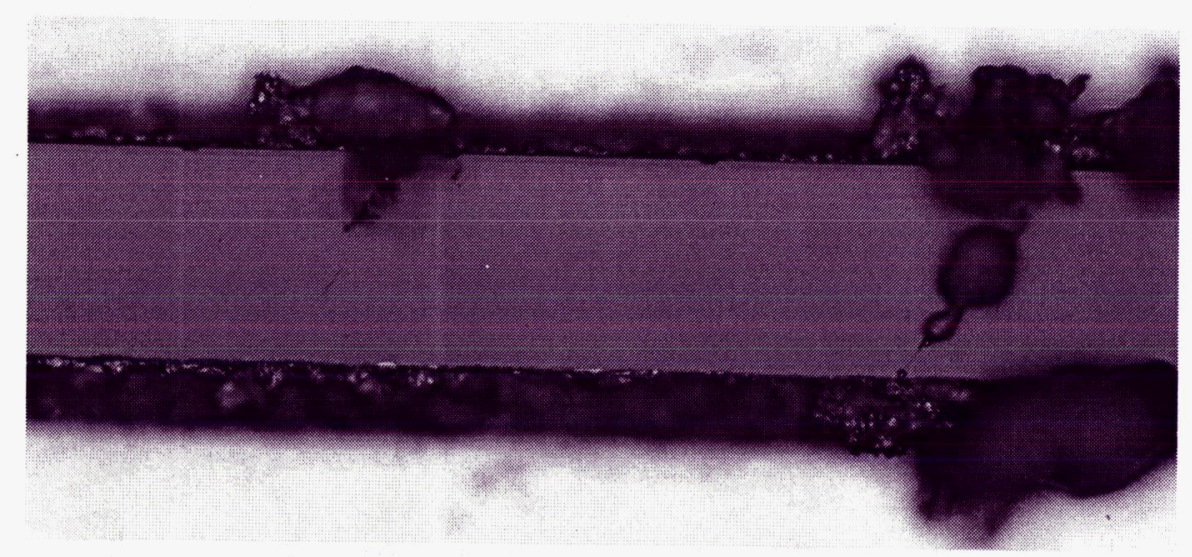

Figure A-3: This figure is a 200X Bright field image of a portion of Device D12, as above. It shows cracks in the facet surface as well as some solder structure which is not well resolved.

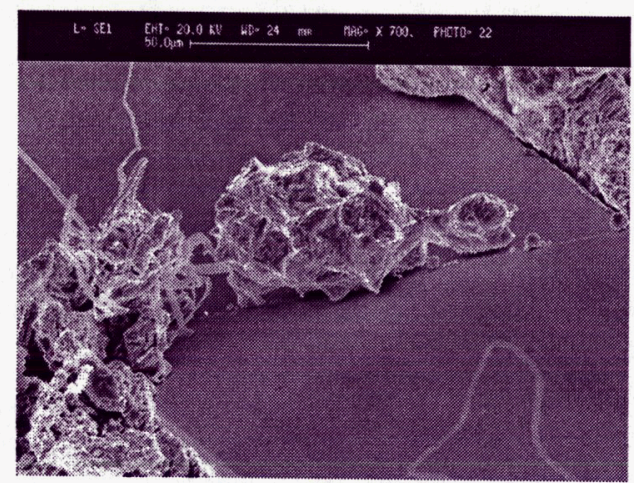

(a)

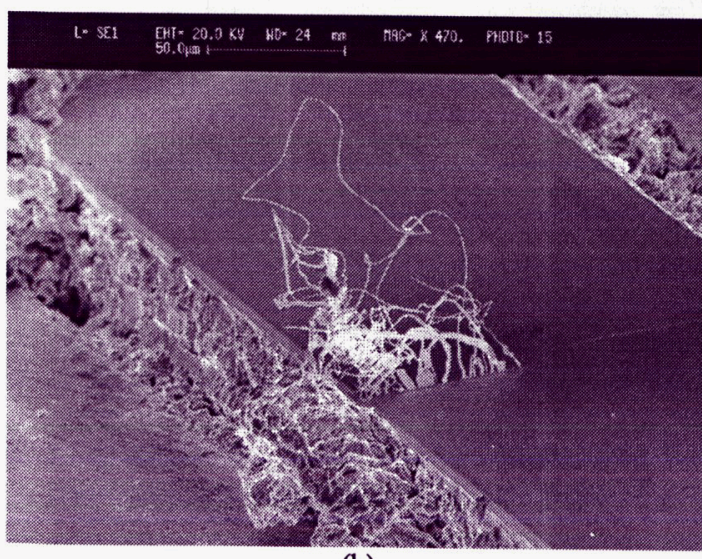

(b)

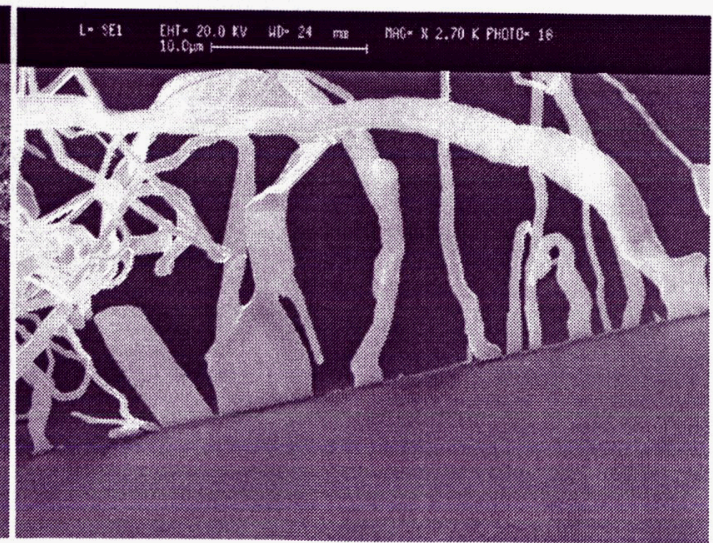

(c)

Figure A-4: This figure shows a scanning electron microscope image of sections of Figure A-3. In (a), the solder feature on the right side of A-3 is resolved. In (b) the facet crack illustrated in the left part of A-3 is illustrated. Portion (c) is a zoomed in section of portion (b) showing the fine structure of the solder being squeezed out from the facet crack. 


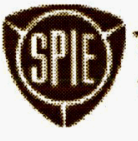

The international Society for Optical Engineering

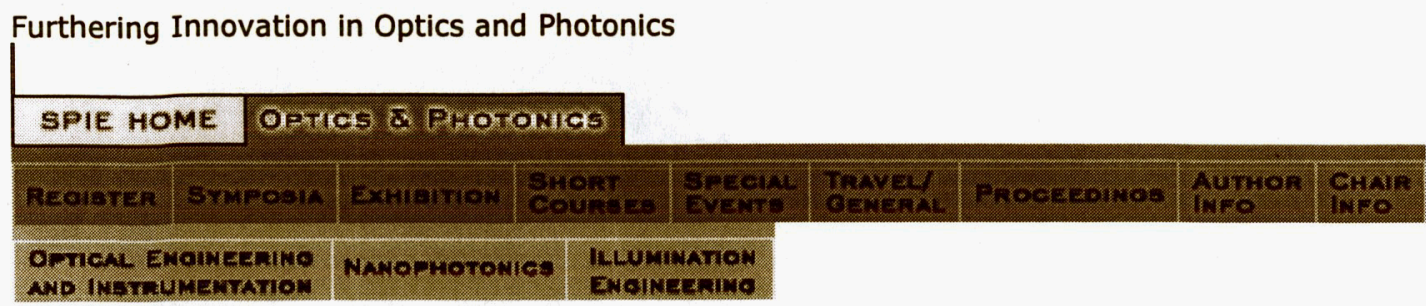

TSrmposia
- Optical Engineering
and Instrumentation
- Nanophotonics
- Illumination
Engineering

\section{DETAILS}

- Participants

- 50 Years of SPIE (150K PDF)

- Printed Advance Program (3.1 MB PDF)

- Printed Call for Papers (1 MB PDF)

- Media \& Publicity

- Letters of Invitation for Visa Process

\section{toprics a photonics}

- Register

- Symposia

- Exhibition

- Short Courses

- Special Events

- Travel/General

- Proceedings

- Author Info

- Chair Info

\begin{tabular}{l} 
SPIEWonks \\
CAREER EXros \\
\hline
\end{tabular}

- Job Seekers

- Employers

\section{Optics \& Photonics 2005 PROGRAM}

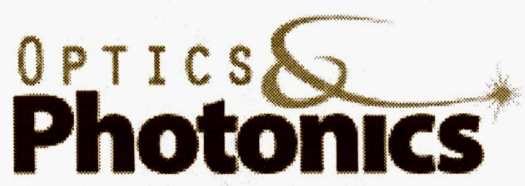

Co-located with the SPIE 50th Annual Meeting

31 July-4 August 2005

San Diego Convention Center

San Diego, California USA

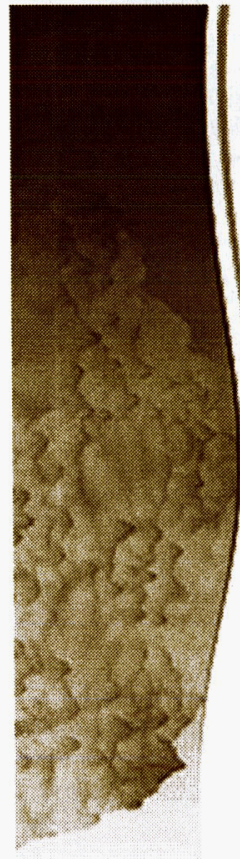

Access the most optics and photonics research available at any event. Featuring over 1,000 presentations on nanotechnology and illumination engineering.

Register today!

\section{Conferences - 70 Courses . 230 Exhibitors}

Optical Engineering and Instrumentation

NanoPhotonics

Illumination Engineering

Honorary Symposium Chairs:

Robert E. Fischer, Optics 1, Inc.

Robert R. Shannon, Optical Sciences Ctr., The Univ. of Arizona

Warren G. Smith, Kaicer Flectro-Optics.

Inc.

Andrew G. Tescher, AGT Associates

Brian J. Thompson, Univ. of Rochester
I PLAN Your WeEk

- Daily Schedules

DIMPORTANT DATES

- Conference Dates

31 July-4 August 2005

- Preregistration: 15 July 2005

- Hotel Reservations by:

28 June 2005

- Manuscripts Due: 5 July 2005

- On-site Manuscripts Due:

9 May 2005

SPPONSORED BY

g(I) The international Society or Optical Engineering

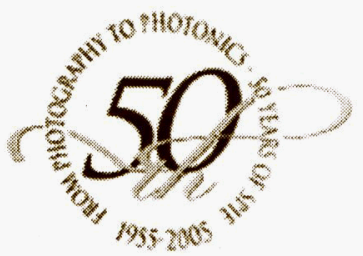


James C. Wyant, Optical Sciences Ctr., The Univ. of Arizona

William L. Wolfe, Optical Sciences Ctr., The Univ. of Arizona

\section{Get the Latest Editor-Reviewed}

Research. . .Faster!

You have options with your registration.

Pick the one that best suits you.

- Fastest: SPIE Digital Library Subscription

- Faster: Printed Proceedings Volumes

- Fast: Searchable CD-ROMs with Multiple Conferences

Things are changing at SPIE, where research is delivered fast.

Cover images are used with permission and can be found in Proceedings of SPIE Vol. 5528, paper 39. C. Chrzanowski, C. Frohlich, G. Shirgur, R. G. Mink authors.

| SPIE Home | Publications | Conferences | Exhibitions | Membership | Education |

Telephone: +1 360/676-3290 | Fax +1 360/647-1445 | Email: spie@spie.org

(C) 1994- 2005 SPIE-The International Society for Optical Engineering

| Privacy Policy |

SPIE is a not-for-profit international society dedicated to advancing optics and photonics. 